\title{
Transient global amnesia: an uncommon presentation of acute myocardial infarction
}

\author{
Paolo Tirelli, ${ }^{1}$ Fulvio Cacciapuoti, ${ }^{2}$ Filomena Scarano, ${ }^{3}$ Federico Cacciapuoti ${ }^{3}$ \\ ${ }^{1}$ Department of Internal Medicine, S. Maria di Loreto Nuovo Hospital; ${ }^{2}$ Emergency Department, A. Cardarelli Hospital; \\ ${ }^{3}$ Department of Internal Medicine, L. Vanvitelli University, Naples, Italy
}

\begin{abstract}
Transient global amnesia (TGA) is an acute neurological syndrome characterized by sudden-onset global (anterograde and retrograde) amnesia, without compromising other neurological functions. This clinical condition lasts up to $24 \mathrm{~h}$ with whole restoration. Several causes have been proposed as responsible for it. Among these, acute myocardial infarction (AMI) was reported as a rare cause of TGA. Sympathetic hyperactivity occurring too early in AMI seems to induce vasospasm, responsible for venous cerebral congestion acting on hippocampal and temporal structures and consequent memory loss. A rare case of TGA as precocious display of subsequent AMI was described.
\end{abstract}

\section{Introduction}

Transient global amnesia (TGA) is a wellrecognized clinical syndrome showing as sudden-onset temporary loss of anterograde and recent retrograde memory and impaired ability to retain new information. It evolves towards a complete recovery of prior neurological status within 24 hours, without compromising the other neurologic functions. ${ }^{1}$ Its pathogenesis is not well known but, venous congestion, migraine, arterial ischemia or epilepsy are proposed as frequent causes of TGA. ${ }^{2}$ Another cause

Correspondence: Federico Cacciapuoti, Internal Medicine Department, L. Vanvitelli University, Naples, Italy.

E-mail: fulviocacciapuoti@gmail.com

Key words: Transient global amnesia; hippocampus; memory loss; acute myocardial infarction; sympathetic hyperactivity.

Contributions: PT and FS found and managed the patient; FC1 contributed to the discussion and found the references; FC2 conceived the paper and wrote the manuscript.

Conflict of interest: the authors declare no potential conflict of interest.

Received for publication: 7 November 2017.

Revision received: 30 January 2018.

Accepted for publication: 8 February 2018.

This work is licensed under a Creative Commons Attribution NonCommercial 4.0 License (CC BY-NC 4.0).

CCopyright P. Tirelli et al., 2018

Licensee PAGEPress, Italy

Italian Journal of Medicine 2018; 12:148-150

doi:10.4081/itjm.2018.945 is an increased sympathetic hyperactivity, likewise to stress (or Takotsubo) cardiomyopathy. ${ }^{3}$

The etiology of TGA generally depends on triggering events, such as sexual intercourse, cold water contact, temperature changes, physical exertion or, seldom, acute myocardial infarction (AMI). In these circumstances, patients suffer from impaired autonomic function characterized by a sympathetic hyperactivity, leading to an increased central venous return, with retrograde high-pressure venous flow to hippocampal region and diencephalic structures. ${ }^{4}$ In fact, clinical and experimental data show that hippocampal neurons are involved in the process of memory. Lesions to this area produce a significant memory impairment that can be responsible for TGA. ${ }^{5}$

In this report, we refer to a case of inferior AMI chiefly displayed as TGA.

\section{Case Report}

A 64-year-old man was admitted to Emergency Department of S. Maria of Loreto Nuovo Hospital for sudden onset of memory loss and confusion. The patient was disoriented in regard to time and place, but he did not remember his name and did not recognize the family members. Besides agitation with repetitive queries, no other symptoms were reported. Upon examination, no focal neurologic signs were detected. Particularly, transient cerebral ischemia, migraine, and epilepsy were excluded but, on the basis of clinical symptoms and negative results of an urgent computed tomography of the brain, a diagnosis of TGA was established. Although the patient did not indicate chest pain at admission, he was subsequently admitted to the Intensive Care Unit for several episodes of angina pectoris, approximately appeared after 8 hours from 
hospital admission. In agreement, cardiologic evaluation, including electrocardiogram (EKG) and dosage of cardiac troponin I, were normal. However, after $12 \mathrm{~h}$, troponin I serum concentration was $=2.5$ $\mathrm{ng} / \mathrm{mL}$ and second EKG disclosed mild ST-segment elevation in D II-DIII-aVF (Figure 1), that led to the diagnosis of inferior ST elevation myocardial infarction. Coronary angiography later performed, disclosed a culprit occlusion of distal part of right coronary artery (Figure 2). Cardiac and clinical postinterventional course was uneventful and the patient was discharged from the hospital on the eighth day after admission.

\section{Discussion and Conclusions}

The incidence of TGA is approximately 3 to 10 people per 100,000 worldwide. It typically appears in patients between 50-80 years of age. Several hypotheses were suggested about the underlying cause of TGA, but none was unwary confirmed. Among these, such as cerebral ischemia, migraine, epilepsy and venous congestion, there has been great deal of conjecture but, at moment, there is no consensus as triggers of TGA. But TGA can also be reported as induced by AMI (acting as trigger of the cerebral syndrome). ${ }^{6}$ In fact, patients with AMI are known to

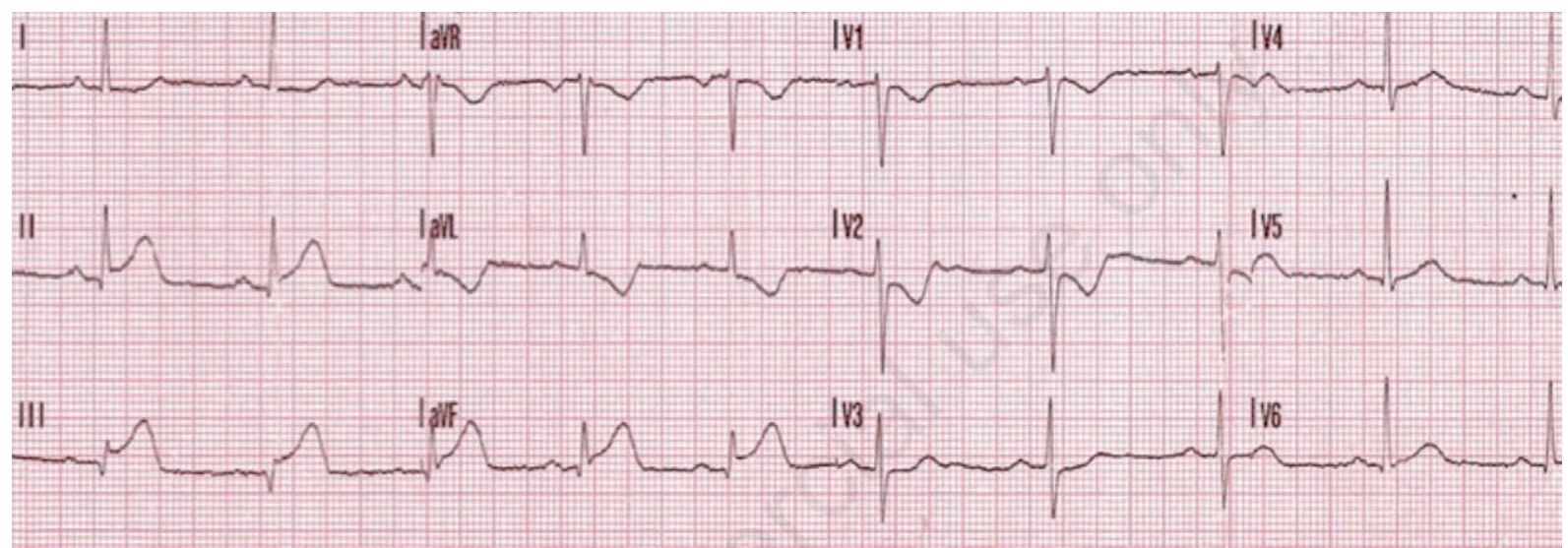

Figure 1. 12 leads electrocardiogram (inferior ST elevation myocardial infarction): S-T tract elevation in DII-DIII-aVF.

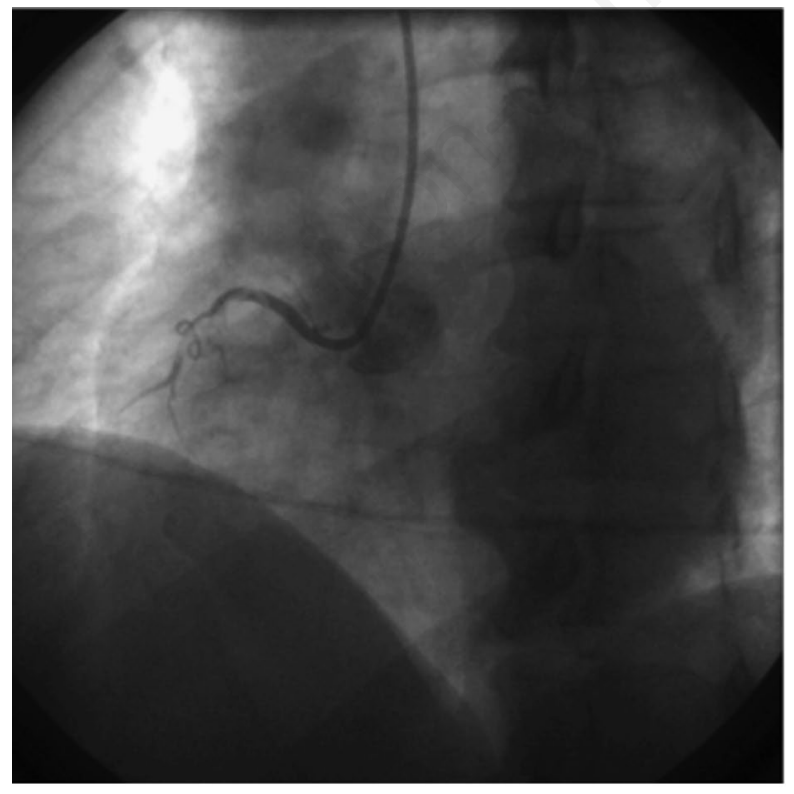

BEFORE

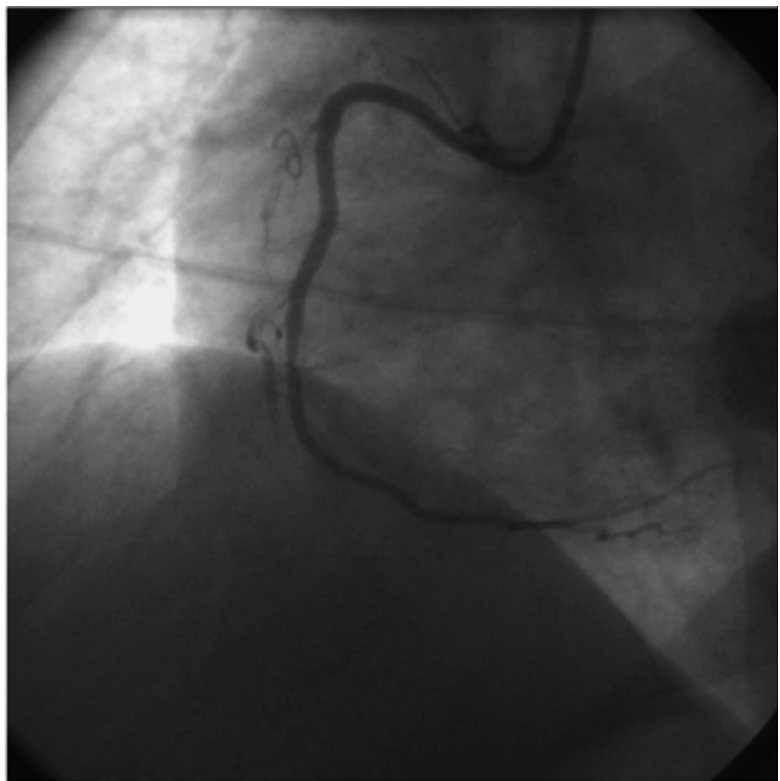

AFTER

Figure 2. Coronary arteriography-proximal occlusion of right coronary artery. Before and after interventional revascularization. 
have impaired autonomic function. In particular, sympathetic hyperactivity has been shown to occur in the early phases of AMI. ${ }^{7}$ Probably TGA during AMI depends on the block of venous return consequent to vasospasm induced by sympathetic hyperactivity that may allow brief retrograde transmission of high venous pressure to the cerebral venous system. In other words, cerebral vasoconstriction can result in changes in the cerebrovascular hemodynamics within the hippocampal region. Venous congestion with retrograde cerebral flow is another hypothesis for the pathophysiology behind TGA. One theory previously proposed by Lewis is that venous congestion causes disrupted blood flow to the thalamic or temporal structures with consequent memory loss. ${ }^{2}$ Subsequently, Baracchini et al. supposed that TGA can be a consequence of a disturbance of venous hemodynamics. ${ }^{8}$ Recently, a unifying hypothesis has been put forward by Winbeck et al. In that, TGA can result from transient ischemia of memory-relevant structures either from an arterial embolus or from venous ischemia. ${ }^{9}$ Actually, among these hypotheses, cerebral venous dysfunction due to vasospasm seems to be more probable, even though it does not completely explain the symptoms of TGA and the other triggering causes were not clearly confirmed. But, the relationship between heart and mind in AMI inducing TGA is a rare and convincing connection between two organs. ${ }^{10}$

\section{References}

1. Arena JE, Rabinstein AA. Transient global ischemia. Mayo Clinic Proc 2015;90:264-72.

2. Lewis SL. Aetiology of transient global ischemia. Lancet 1998;352:397-9.

3. Finsterer J, Stollberger C. Transient global ischemia: the cerebral Takotsubo? J Neurol Sci 2017;376:196-7.

4. Agosti C, Borroni B, Akkavi NM, et al. Acute myocardial infarction presenting with transient global amnesia. Am Geriatrics Soc 2006;54:1004-23.

5. Bartsch T, Alfke K, Deuschi G, Jansen O. Evolution of hippocampal CA-1 diffusion lesions in transient global amnesia. Ann Neurol 2007;62:475-80.

6. Courand PY, Sibellas F, Gonidec S, et al. Acute myocardial infarction: a precipitating event for transient global amnesia. J Cardiovasc Med 2013;15:78-9.

7. Graham LN, Smith PA, Hugger RJ, et al. Simpathetic drive in anterior and inferior uncomplicated acute myocardial infarction. Circulation 2004;109:2285-89.

8. Baracchini C, Tonello S, Farina F, et al. Jugular veins in transient global amnesia: innocent bystanders. Stroke 2012;43:2289-92.

9. Winbeck K, Etgen T, van Einsiedel HG, et al. DWI in transient global amnesia and TIA: proposal for an ischemic origin of TGA. J Neurol Neurosurg Psychiatr 2005;76:438-41.

10. Jain P, Michel J. McCrohon J. Of hearts and minds: a case of simultaneous transient global amnesia and regional left ventricular dysfunction. Int Journ Cardiol 2015;198:49-50. 